\title{
Sexueller Missbrauch in der katholischen Kirche als Skandal
}

\author{
Oder: Wie und warum sich die Grenzen des Sagbaren verschieben
}

\author{
Thomas Großbölting
}

„Katholische Nadelstiche gegen Nazis“ - unter dieser Überschrift veröffentlichte die in Oldenburg i. O. erscheinende Nordwest-Zeitung am 21. Januar 2021 einen Artikel über den ehemaligen Kaplan Georg Meyer in Markhausen. Darin berichtete der zuständige Lokalredakteur, wie sich der später über viele Jahrzehnte in der Gemeinde engagierte, 1970 verstorbene Geistliche in seinen jungen Jahren gegen örtliche Nationalsozialisten gestemmt hatte. Sein mutiges Verhalten brachte ihm posthum sogar eine Straßenwidmung in der nahegelegenen Bauernschaft Sedelsberg ein - die "Kaplan-Meyer-Straße“ erinnerte an ihn. So weit - so gewöhnlich: Im oldenburgischen Münsterland als Wirkungskreis des Kardinals Graf von Galen gehören derartige Verweise bis heute zum immer wieder aktualisierten Traditionsbestand des Katholizismus.

Aber diesmal war doch etwas anders - das Geschriebene traf nämlich nicht nur auf die sonst übliche selbstbestätigende wohlwollend-desinteressierte Kenntnisnahme. Ein Satz hatte einen Leser so tief bewegt, dass er weder den Bericht noch die Ehrung unkommentiert lassen wollte: Gelobt worden war der Geistliche unter anderem dafür, dass er "durch besondere Methoden die Jugendlichen zu sich herübergezogen hatte“. Genau diese Bemerkung, so der anonym bleibende Leser, „lässt alles in mir hochkommen." Als Kind habe Meyer ihn mehrmals sexuell missbraucht. Der Geistliche habe ihn in die Sakristei gezogen, an sich gedrückt und versucht, ihm seine Zunge in den Mund zu schieben. Er selbst habe weitergehende Berührungen vermeiden können, aber „von anderen Jungs wurde mir erzählt, dass es bei den Messdienern in der Sakristei zu schweren sexuellen Übergriffen gekommen ist." ${ }^{1}$

Die nach diesem Leserbrief einsetzende Entwicklung zeigt vor allem eines: Im Ort waren diese und andere Vorfälle weithin bekannt. Meyer hatte zwar seinerzeit die Jungen zur Verschwiegenheit verpflichtet; auch deshalb trauten sich die Betroffenen selbst nicht oder nur im kleinsten Kreis, über das ihnen angetane Leid zu sprechen. Trotzdem wussten viele über den Kreis der Betroffenen hinaus, dass Meyer über viele Jahre Jungen aus dem Kreis der Messdiener intensiv missbraucht hatte. In der Gemeinde erzählt man sich

1 Carsten Bickschlag, Schwere Vorwürfe gegen Pfarrer, in: Nordwest-Zeitung, 13.2.2021, 10. 
heute, dass insbesondere die Dorfeliten ihre Söhne vom Ministrantendienst ferngehalten hätten. Abgesehen davon aber schwieg man und ließ den übergriffigen Geistlichen gewähren. Die eng gezogenen Grenzen des Sagbaren und seine Machtstellung im Gemeinde- und Sozialgefüge sollten ihn noch über Jahrzehnte schützen.

Erst die Reaktionen auf die Enthüllungen und Berichte in der Presse am Anfang des Jahres 2021 brachen das kollektive Schweigen, und das mehr als ein halbes Jahrhundert nach den Taten. In der neu entstandenen Öffentlichkeit stellte sich heraus, dass sich bereits 2010 ein anderer Betroffener beim Bistum Münster gemeldet hatte. In einem Verfahren zur Anerkennung seines Leids war ihm ein Geldbetrag zugesprochen worden - ein Hinweis darauf, dass die Verantwortlichen die Aussagen des Mannes als glaubhaft einstuften. Im Februar und März 2021 meldeten sich dann im Münsteraner Forschungsprojekt zur Aufarbeitung des Missbrauchs im Bistum Münster weitere Betroffene. Die aussagekräftigen und glaubwürdigen Zeugnisse deuten aktuell auf mindestens sieben Personen hin, die eigenen Angaben zufolge oder gemäß Hinweisen von Familienangehörigen durch Kaplan Meyer sexuell missbraucht worden waren. Die Taten erstreckten sich über die Jahre 1954 bis 1968; im Einzelnen missbrauchte er seine Opfer über Zeiträume zwischen einem und fünfJahren. Auch wenn all diese Angaben letztlich keinen juristischen Schuldspruch ersetzen, so verdichten sie sich bis hin zur Gewissheit. Die über viele Jahrzehnte so massiven Grenzen des Sagbaren waren offenbar innerhalb weniger Wochen gefallen. Die Kirchengemeinde und die örtliche Kommune sind alarmiert, stellen sich doch Fragen nach der Verantwortung und der Schuld weit über den Geistlichen hinaus. Wie soll sich das Zusammenleben jetzt und in Zukunft gestalten? Im März 2021 beschlossen Lokalpolitikerinnen und -politiker die Umbenennung des nach dem mutmaßlichen Missbrauchstäter benannten Weges: Die „Kaplan-Meyer-Straße“ heißt seitdem „Zur Sporthalle“. ${ }^{2}$

In seinen spezifischen lokalen Kontexten ist der Fall Meyer sicher besonders - und steht dennoch in einer Reihe von vielen, mittlerweile auch dokumentierten und veröffentlichten Beispielen. Exemplarisch soll nur ein weiteres Vorkommnis kurz skizziert werden, um die erstaunlichen Parallelen

2 Ders., Neuer Straßenname in Sedelsberg so grau wie das Pflaster, in: NWZonline, 26.3.2021, einzusehen unter https://www.nwzonline.de/plus-cloppenburg-kreis/sedelsberg-umbenen nung-nach-missbrauchsskandal-neuer-strassenname-in-sedelsberg-so-grau-wie-daspflaster_a_51,0,2484410637.html, zuletzt aufgerufen am 14.4.2021; Heiner Stix, Missbrauchsvorwürfe gegen Pfarrer in Markhausen: Forderung nach Straßenumbenennung wird laut, in: OM online, 16.2.2021, einzusehen unter https://www.om-online.de/politik/ missbrauchsvorwurfe-gegen-pfarrer-in-markhausen-forderung-nach-strasenumbenennungwird-laut-63014, zuletzt aufgerufen am 24.3.2021. 
zu dokumentieren: Im Herbst 1994 sollte die Haupt- und Realschule im niedersächsischen Neuenkirchen nach dem langjährigen Pfarrer Bernhard Janzen benannt werden. Der Geistliche wurde ein halbes Jahr vor seinem Tod 1972 mit einer Ehrenbürgerschaft ausgezeichnet, als Dank für seine Verdienste um den Schulausbau, die Klinikgründung und den sozialen Wohnungsbau in der ländlichen Gemeinde. Es war dann ein Opfer von Janzen, das die an ihm verübten Missbrauchstaten öffentlich machte. Bis dahin hatte Bernd Theilmann nicht darüber gesprochen, was der Pfarrer ihm angetan hatte: „Wenn Du einem davon erzählst, dann tritt der Satan zwischen uns!", so hatte Janzen Theilmann gegenüber gedroht. Wer die Auswüchse katholischer Strafpastoral kennt, der wird ermessen können, welche Wucht ein solcher Satz gegenüber einem kleinen Jungen entfalten kann. Theilmann jedenfalls rüttelt es noch heute durch, wenn er daran zurückdenkt: „,Wie er das sagte: ,Der Satan'... Theilmann zischt das S, es klingt wie bei einer Schlange.“3

Er brach 1994 sein Schweigen, erzählte seinen Eltern vom Geschehen im Pfarrbüro und informierte im zweiten Schritt den Bürgermeister. Dieser wiederum sprach den örtlichen Pfarrer an. Es passierte - nichts. Der Gemeinderat, der über die Vorwürfe nicht informiert war, stimmte mit großer Mehrheit dafür, die Schule nach dem Ehrenbürger Janzen zu benennen. Theilmann akzeptierte das erneute Schweigen nicht, ging an die Presse. Auch in diesem Fall diskutierte die örtliche Politik - allerdings nicht über den Missbrauch an sich, sondern über das Agieren des Bürgermeisters. Warum hat er nicht die politischen Gremien informiert? Der Gemeindeobere verteidigte sich mit dem Hinweis, dass es sich um einen "diffusen und vor allem nicht nachvollziehbaren Vorwurf" gehandelt habe. ${ }^{4}$ Andere, die sich an der öffentlichen Debatte beteiligten, beschimpften nun die Opfer: „Sind sich die jungen Männer dessen bewußt, was sie ihrer Heimatgemeinde angetan haben?", fragte in einem Leserbrief ein Ehepaar, welches vorgab, „im Namen vieler“ zu sprechen. Erst 2010 stellte eine Kommission von Fachleuten aus Wissenschaft, Justiz und Verwaltung „mit moralischer Gewissheit“ fest, dass „Pfr. Bernhard Janzen Kinder, Jugendliche und junge Erwachsene sexuell missbraucht hat. ${ }^{45}$

Die Grenzen des Sagbaren - das machen diese beiden wie auch viele ähnlich gelagerte Fälle deutlich - waren bis in die 199oer Jahre und auch darüber hinaus eng gezogen. Thematisiert wurde der Missbrauch allenfalls in Andeutungen. „Zu dem geh' lieber nicht allein“, so hatten sich beispielsweise

3 Karsten Krogmann, Das Schweigen der Gemeinden, in: Forum Opferhilfe 43(3), 2020, 5-10, hier 7 .

4 Zit. n. ebd., 9.

5 Zit. n. ebd., 10. 
die Jugendlichen auf dem Spielplatz gegenseitig vor Pfarrer Janzen gewarnt. ${ }^{6}$ "Jetzt ist der schwule Bock endlich weg!", tönte es auf dem Schulhof nach der Versetzung des Pfarrers Helmut Behrens, der in Neuscharrel zahlreiche Kinder missbraucht hatte. "Messdienerwitze“ über Geistliche und deren sexuelle „Vorlieben“ machten die Runde zusätzlich zu den schon gängigen Zoten über Pfarrer mit ihren Haushälterinnen. Unabhängig von ihrem jeweiligen ästhetischen Wert funktionieren diese Witze subversiv wie auch entlastend als „ersparter Hemmungsaufwand“ (Sigmund Freud). Gerade diese Form des Humors ermöglichte es, das aufzugreifen, was zu sagen eigentlich nicht erlaubt oder zumindest nicht opportun war oder ist.

Die große Mehrzahl der heute diskutierten Fälle von sexuellem Missbrauch ereignete sich zwischen $195^{\circ}$ und 1990, öffentlich bekannt und skandalisiert wurden sie erst viele Jahre später. Durchschnittlich dauerte das Schweigen der Betroffenen mehr als 25 Jahre. ${ }^{8}$ Allein diese Zahl macht deutlich, wie viel Mut es vonseiten der Betroffenen bedurfte, um die Missbrauchs- und Gewalttaten öffentlich zu machen und damit zum Skandal werden zu lassen.

Ein Wendepunkt war bezeichnenderweise nicht die Aufdeckung der Missbrauchstaten im internationalen (vor allem im US-amerikanischen) Katholizismus, sondern der „Fall“ des Berliner Canisius-Kolleg. Im Januar 2010 hatte der damalige Schuldirektor und Jesuit Klaus Mertes an 6oo Ehemalige geschrieben und ihnen mitgeteilt, was er selbst von drei früheren Schülern erfahren hatte: Zwei Jesuitenpatres, die an der Schule unterrichteten, hatten sie und andere Schüler in den 197oer Jahren sexuell missbraucht. Mertes' Initiative ermutigte auch andere Betroffene, sich zu melden und das ihnen angetane Unrecht öffentlich zu machen. Erstmalig wurde damit auf breiter Basis Missbrauch in der bundesdeutschen Öffentlichkeit skandalisiert. Erst seitdem scheinen die bis dahin eng gezogenen Grenzen des Sagbaren beim Thema sexueller Missbrauch durchbrochen. Für viele Betroffene in anderen Zusammenhängen hatte das eine ähnliche Signalwirkung wie die 2014 beauftragte und 2018 abgeschlossene MHG-Studie, die den „sexuellen Missbrauch an Minderjährigen durch katholische Priester, Diakone und männliche Ordensangehörige im Bereich der Deutschen Bischofskonferenz" umfassend aufarbeitete. ${ }^{9}$ Die Missbrauchstaten können veröffentlicht, skandalisiert und damit auch politisch-gesellschaftlich bearbeitet werden.

6 Zit. n. ebd., 8.

7 Zit. n. ebd.

8 Mary Hallay-Witte/Bettina Janssen (Hrsg.), Schweigebruch. Vom sexuellen Missbrauch zur institutionellen Prävention. Freiburg im Breisgau/Basel/Wien 2016, 102.

9 Harald Dreßing u.a., Sexueller Missbrauch an Minderjährigen durch katholische Priester, Diakone und männliche Ordensangehörige im Bereich der Deutschen Bischofskonferenz, 
Dennoch sind die Grenzen des Sagbaren, die die Aufdeckung vergangener und aktueller Fälle verhindern, weiterhin vorhanden. Oft bleiben eine hohe Schwelle persönlicher Scham und Betroffenheit wie auch die Angst vor möglichen Zweifeln und Abwehrreaktionen, die das Reden über den Missbrauch nach wie vor zu einem Wagnis machen. Im Folgenden soll die damit verbundene Frage aufgegriffen werden, warum es heute vor allem für die Betroffenen, aber auch für die Öffentlichkeit insgesamt einfacher ist und dennoch schwierig bleibt, Missbrauch zu thematisieren und die Unrechtstaten zu skandalisieren.

\section{Skandalisierung: Wissen und Macht in der Thematisierung von sexuellem Missbrauch}

Abstrakt gesprochen liegen der Dynamik der beiden eingangs skizzierten Fallgeschichten ähnliche Zusammenhänge und Mechanismen zugrunde: Ein Skandal ist eine Normverfehlung oder - wie im Fall des sexuellen Missbrauchs ein von einer Person, einer Gruppe oder einer Institution begangenes Delikt, welches dann tatsächlich ans Licht kommt, öffentlich bekannt und infolgedessen zum Gegenstand öffentlicher Empörung wird. ${ }^{10}$ Erst wenn genügend Aufmerksamkeit erreicht ist, kann es gelingen, eine in dem Sinne produktive Diskussion zu starten, dass die Täter bestraft, Wiedergutmachungen so weit wie möglich geleistet und das Normengefüge der Gesellschaft, vielleicht sogar die rechtlichen Bedingungen neu ausgerichtet werden.

Die Analyse von Skandalen legt deshalb nicht nur Normbrüche offen, sondern lässt viele weitere Facetten hervortreten: Zuerst entwickeln sich in Skandalen gesellschaftliche Wertvorstellungen und Normenkomplexe, die in der Skandalisierung thematisiert und bearbeitet werden. Skandale und ihre Dynamik lassen erkennen, wie kritische Öffentlichkeit entsteht, wer diese konstituiert und wie sie strukturiert ist. Skandale lassen auch die (vormals) bestehenden Grenzen des Sagbaren hervortreten und zeigen damit, welchen Machtstrukturen das Wissen darum unterlag.

Verbrechen wie auch Normbrüche anderer Art werden, so die Grundvoraussetzung, erst dann zum Skandal, wenn sie eine gewisse Öffentlichkeit erlangen. Das bedeutet nicht, dass die inkriminierten Taten vorher geheim waren. Gerade

Mannheim/Heidelberg/Gießen, 24.9.2018, einzusehen unter https://bistumlimburg.de/ fileadmin/redaktion/Bereiche/missbrauch/MHG-Studie-gesamt.pdf, zuletzt aufgerufen am 5.5.2021.

10 Frank Bösch, Kampf um Normen. Skandale in historischer Perspektive, in: Kristin Bulkow/ Christer Petersen (Hrsg.), Skandale. Strukturen und Strategien öffentlicher Aufmerksamkeitserzeugung. Wiesbaden 2011, 29-48, hier 33 . 
das Beispiel sexueller Missbrauch im katholischen Zusammenhang bietet eindrucksvolle Beispiele für das Gegenteil: Im Fall der Regensburger Domspatzen gab es im Jahr 1989 Berichte in der lokalen und regionalen Presse, die Gewalttaten und sexuellen Missbrauch im Chor öffentlich machten. Nur: Eine Skandalisierung erfolgte nicht. Hatte vier Jahrzehnte zuvor noch die unmittelbare Intervention von Spitzenleuten des Bistums eine weitere Berichterstattung verhindert - 1946 warnte der damals einflussreiche Münchner Weihbischof Neuhäusler vor „Verleumdungen, Lausbübereien und Verbrechen“ - waren es in diesem Fall, so vermuten die Historiker Bernhard Löffler und Bernhard Frings, die Ereignisse um die deutsche Wiedervereinigung, die den Missbrauch in Regensburg in den Hintergrund rücken ließen." ${ }^{11}$ Die Domspatzen waren als "bayerisches Kulturgut" ein perfekter Werbeträger für das Bistum ebenso wie für das Land Bayern, die Domkapellmeister Stars ihrer Zunft, deren Können nicht zuletzt durch die internationalen Auftritte und die dabei gewonnene Reputation des Chores bestätigt wurden. Ein kompliziertes Geflecht aus Trägerstrukturen und einer hauptsächlich auf den jeweiligen starken Mann zugeschnittene Organisationsstruktur bescherte dem jeweiligen Domkapellmeister eine starke Machtposition, die auch Justiz und Polizei davor zurückschrecken ließen, den Missbrauchstaten offensiv nachzugehen. Frings und Löffler resümieren: „Fast 65 Jahre lang entstand aus diesen Kenntnissen [...] kein wirklicher, Konsequenzen einfordernder Außendruck, der zum Eklat geführt hätte."12

Als sich 1989 ein ehemaliges Mitglied der Domspatzen vor einem Münchener Gericht wegen versuchten Mordes und gefährlicher Körperverletzung verantworten musste, änderte sich die Zurückhaltung wenigstens der Justiz, ohne dass die Vorgänge eine darüber hinausreichende größere Aufmerksamkeit erhielten. Der frühere Chorsänger entschuldigte seine Taten mit einer Traumatisierung, die darauf zurückzuführen sei, dass er im Regensburger Internat selbst Opfer von gewalttätigen, zum Teil wohl auch sadistischen Praktiken geworden sei.

Erst 2010 aber und speziell im Zusammenhang mit der Aufdeckung der Missbrauchstaten im Canisius-Kolleg in Berlin platzte der Knoten. Die Vorwürfe gegen die Regensburger wurden seit diesem Zeitpunkt in die Szene bundesweit und weltweit zu beobachtender Missbrauchsfälle eingeordnet. ${ }^{13}$

11 Bernhard Frings/Bernhard Löffler, Der Chor zuerst. Institutionelle Strukturen und erzieherische Praxis der Regensburger Domspatzen 1945 bis 1995. Regensburg 2019, 317-320.

12 Ebd., 301.

13 Siehe dazu den Beitrag von Wim Damberg im vorliegenden Band. 
Wenn über viele Jahrzehnte hinweg die Autorität und das Sozialprestige einer kirchlichen Einrichtung, wie auch eines einzelnen Klerikers, in vielen Zusammenhängen solche Taten (mit)begünstigten und den Aufklärungswillen überwogen, dann ging diese Gleichung seit den 199oer Jahren nicht mehr auf. Mit dieser Veränderung setzte ein Aufarbeitungsprozess ein und gewann eine Dynamik, die bis heute anhält.

Eine frappierende, sicher auch erschreckende Erkenntnis aus der Aufarbeitung von Missbrauch in der katholischen Kirche ist das hohe Maß an explizitem, vor allem aber implizitem internen Wissen lange vor der öffentlichen Thematisierung der Taten: Nicht allein die hoch unprofessionell geführten Personal- und Sachakten in den Bistümern selbst offenbaren dieses trotz ihrer euphemisierenden Sprache (Der Kölner Kardinal Joachim Meißner etwa beschriftete die von ihm über Missbrauchstaten von Klerikern geführte Akte mit „Brüder im Nebel ${ }^{414}$ ). Die Gerüchteküche brodelte, und mindestens implizit, gelegentlich auch explizit waren auch sexuelle Verfehlungen ein Thema. Im Priesterseminar, unter den Mitgliedern der verschiedenen Weihejahrgänge, kannte man sich und wusste voneinander. Ebenso waren und sind Kirchengemeinden und Nachbarschaften Soziotope mit einem dichten gemeinsamen Wissensstand. Die Grenzen des Sagbaren wurden dann oftmals anonym durchbrochen. Im Fall des äußerst populären Kaplans Theo Wehren in Bocholt-Barlo, dessen Spitzname "Kapi“ sogar für die Benennung eines Spielplatzes herangezogen wurde, waren es beispielsweise Zettel am Grabstein eines verstorbenen Pfarrers, die subversiv wirkten und das Schweigen brachen..$^{15}$ Gab es unter Klerikern Netzwerke des Missbrauchs, in denen sich einzelne Priester unterstützten und beispielsweise Opfer zuführten? Was in Betroffeneninterviews gelegentlich thematisiert wird, bleibt empirisch eine offene Frage.

Skandale sind „Orte der Konfrontation“, an denen Machtstrukturen aufeinanderprallen. So wie der Missbrauch selbst vor allem als Übermächtigung beschrieben werden kann, in der der Täter seinem Opfer die physische oder psychische Integrität nimmt, so ist auch die Skandalisierung des Missbrauchs vor allem eine Machtfrage. Beziehungsgeflechte und Hierarchien werden neu konstituiert. Es wird ausgehandelt und neu entschieden, was als „normal“ gilt. Auf diese Weise sind Skandale nicht anrüchig oder schlecht, sondern

14 Raoul Löbbert, Brüder im Nebel, in: Die Zeit, 18.3.2021, einzusehen unter https://www. zeit.de/gesellschaft/zeitgeschehen/2021-o3/katholische-kirche-gutachten-sexuellermissbrauch-erzbistum-koeln-kardinal-woelki, zuletzt aufgerufen am 7.4.2021.

15 Horst Andresen, Barlo geschockt über „Kapis“ dunkle Seite, in: Westfälische Nachrichten, 3.7.2019, einzusehen unter https:/www.wn.de/Muensterland/3858168-Missbrauch-Barlo -geschockt-ueber-Kapis-dunkle-Seite, zuletzt aufgerufen am 31.3.2021. 
hochproduktiv: Sie helfen dabei, Gesellschaften ins Gespräch mit sich selbst zu bringen und Normsetzungen weiterzuentwickeln und durchzusetzen. Skandale unterlaufen Macht, indem sie vormals toleriertes, akzeptiertes oder zumindest nicht sanktioniertes Fehlverhalten aufgreifen, zum Gegenstand der Empörung machen und mit der dann einsetzenden Neubewertung neue Machtstrukturen schaffen. ${ }^{16}$

Wie kein anderer hat der französische Sozialphilosoph Michel Foucault mit seinen diskurshistorischen Überlegungen zu "Sexualität und Wahrheit“ dazu beigetragen, Skandale zu verorten. ${ }^{17}$ Besonders skandalträchtig sind Ereignisse demnach, wenn sich in ihnen das „Werden eines Wissens“ mit der „Lust, die Lust zu wissen" verbindet: Sexualität in ihren unterschiedlichen Formen war immer ein Feld der Skandalisierung, bei dem sich in der Neuzeit neue Strukturen von Wissen und Macht mit Voyeurismus verbanden. ${ }^{18}$ Umgekehrt trifft die Skandalisierung dann besonders leicht auf Resonanz, wenn das Objekt des Skandals nach außen hin einen besonders hohen Anspruch vermittelt hatte - und sich genau jetzt in dieser Hinsicht verfehlte. Skandale betreffen in diesem Sinne nicht nur die unmittelbar Beteiligten, sondern sind vor allem ein Medium darüber hinaus an die Zuschauenden.

Skandale sind Produkte und Promotor von Veränderungen in der gesellschaftlichen Wertehierarchie. ${ }^{19}$ Blickt man mit dieser Perspektive auf den sexuellen Missbrauch, dann wirken die Grenzen des Sagbaren weit über die Regulierung von Sprachgewohnheiten hinaus. Sie sind vielmehr unmittelbar mit der Praxis des Missbrauchs verbunden. Wie das Missbrauchsgeschehen selbst, lässt sich das Schweigen und das Reden über Missbrauch vor allem unter dem Rubrum von Macht beschreiben: Wie im gesellschaftlich-politischen Diskurs Deutungshoheit entsteht, wer Aufmerksamkeit für seine Inhalte, Ideen und Thesen generieren kann, wer hingegen mit seinen Positionen ungehört oder doch im Hintergrund bleibt - all das hat sich mit Blick auf Religion und Kirche in der Bundesrepublik und das wiedervereinigte Deutschland massiv verändert.

Wir wissen nicht genau, ob die katholische Kirche und ihre Seelsorgebezüge tatsächlich der oder ein Hotspot von sexuellem Missbrauch sind, also einen Zusammenhang bieten, in dem sich eine Kultur des Missbrauchs, des

\footnotetext{
16 Dies ließe sich in Anlehnung an Foucault formulieren, der zwar selbst nicht über Skandale schreibt, wohl aber Mechanismen zu dessen Verortung entwickelt. Michel Foucault, Sexualität und Wahrheit. Bd. 1: Der Wille zum Wissen. Frankfurt a. M. 1997.

17 Ebd., 7f. passim; Frank Bösch, Öffentliche Geheimnisse. Skandale, Politik und Medien in Deutschland und Großbritannien 1880-1914. München 2009, 13-15.

18 Foucault, Sexualität, 97.

19 Bösch, Geheimnisse, 4.
} 
Wegschauens und des Vertuschens besonders extrem entwickelt hat. Wo sexueller Missbrauch vorkommt und wie oft er geschieht, also zur Inzidenz wie auch zur Prävalenz sexueller Übergriffe in der Bevölkerung, „bestehen für Deutschland keine gesicherten Angaben“. ${ }^{20}$ Manche Ergebnisse aus der Medizin, der Psychotherapie und der einschlägig beschäftigten Pädagogik deuten entgegen der öffentlichen Wahrnehmung darauf hin, dass nicht der Beichtstuhl, die Messdienergruppe oder die kirchliche Jugendfahrt, sondern nach wie vor die Familie der am stärksten von Missbrauch betroffene Zusammenhang ist. ${ }^{21}$ Wegen der starken Tabuisierung und der enorm hohen Dunkelziffer sind Aussagen zu den Tatzusammenhängen erstens rar und zweitens immer mit einem hohen Grad von Vorläufigkeit versehen.

Dennoch konzentriert sich momentan die Diskussion auf den sexuellen Missbrauch in der katholischen Kirche - und das mit einer ganzen Bandbreite guter Gründe: Während auf der einen Seite des Spektrums der enorm hohe Selbstanspruch im Umgang mit Sexualität steht, ist es auf der anderen Seite die Möglichkeit insbesondere für Betroffene, jetzt das ihnen angetane Leid zu thematisieren. Über viele Jahrzehnte war die Skandalisierung der Unrechtstaten blockiert. In den Nachkriegsjahrzehnten verfügte die katholische Kirche als Institution über eine massive Diskursmacht, der einzelne Kleriker über eine davon, aber auch von seiner besonderen sakralen Stellung abgeleiteten Pastoralmacht. Beide Machtkonstellationen ermöglichten den Missbrauch, verhinderten dessen Aufdeckung und begünstigten dessen Vertuschung.

\section{Diskursmacht im deutschen Katholizismus}

Nach 1945 starteten weite Teile der Eliten mit der Perspektive einer umfassenden „Rechristianisierung“ der deutschen Gesellschaft. Als „Siegerin in Trümmern" avancierten beide Kirchen zu Garanten des Neuaufbaus. Auf der Folie der allgemeinen materiellen Bedrängnis, der Desorientierung und des erfahrenen Zivilisationsbruchs stach insbesondere die katholische Kirche hervor. Im Selbstverständnis wie auch in der Außenwahrnehmung galt sie als vom Nationalsozialismus nicht korrumpiert. $\mathrm{Zu}$ einem beträchtlichen Teil hatte sie es zwischen 1933 und 1945 verstanden, eigene Strukturen zu bewahren

20 Andreas Jud, Sexueller Kindesmissbrauch - Begriffe, Definitionen und Häufigkeiten, in: Jörg M. Fegert u.a. (Hrsg.), Sexueller Missbrauch von Kindern und Jugendlichen. Ein Handbuch zur Prävention und Intervention für Fachkräfte im medizinischen, psychotherapeutischen und pädagogischen Bereich. Berlin 2015, 41-49, hier 46.

Ebd. 
und die in ihr versammelten Gläubigen an sich zu binden. Auch wenn man weder in der Hierarchie noch unter den Gläubigen von einem konsequenten Sichverweigern sprechen kann, dominierte nach dem Krieg der Eindruck von Standhaftigkeit und Widersetzlichkeit gegen die NS-Diktatur. Erst mit den 196oer Jahren sollte diese teilweise geschönte Sicht zum Anlass heftiger kircheninterner und öffentlicher Debatten werden.

Da die katholische Kirche im Unterschied zu der evangelischen in der öffentlichen Meinung als nichtnazifizierte Institution galt, kam ihr nicht nur in der deutschen Bevölkerung besondere Autorität zu. Unter den wenigen Deutschen, die die alliierten Besatzungsoffiziere als Gesprächs- und Verhandlungspartner akzeptierten, befanden sich viele katholische Geistliche und Würdenträger. Wie den evangelischen Landeskirchen war auch der katholischen Kirche die Fortsetzung ihrer Arbeit ohne Einschränkungen erlaubt worden. Damit waren beide christlichen Konfessionen gegenüber anderen gesellschaftlichen Großorganisationen wie Parteien oder Gewerkschaften deutlich bevorzugt.

MitdiesemhohenSozialprestige startete insbesondere diekatholische Kirche in die Bundesrepublik und konnte ihre besondere Stellung auch in das neu zu organisierende Staatsgefüge einbringen: „Sozialpartnerschaft“ , „Föderalismus“, „Europa“ und „Subsidiarität" - viele der politischen und gesellschaftlichen Grundpfeiler waren in der katholischen Naturrechts-, Gesellschafts- und Staatstheorie bereits vor- oder zumindest angedacht worden. ${ }^{22}$ Ein indirekter Beleg, die diesen Einfluss summarisch fasst, ist die Kritik des nach Kriegsende wohl prominentesten Protestanten Martin Niemöller. Die Bundesrepublik, so monierte der Kirchenpräsident der evangelischen Kirche Hessen und Nassau 1950, sei ein Gebilde, das "in Rom gezeugt und in Washington geboren“ worden sei. Er verwies damit in einer für die Protestanten damals typischen „nationalen“ Sichtweise auf den in seinen Augen ungebührlich großen Einfluss der Katholiken. ${ }^{23}$

Diese Position setzte sich unmittelbar in Diskursmacht um: Die katholische Kirche gab in den unterschiedlichsten Bereichen Standards von Verhalten und Moral vor, auch wenn sich diese aus ihren ursprünglich christlichen Bezügen längst gelöst hatten. Sie war nicht nur eine gewichtige Stimme, wenn es um Familie, die Rollenbilder von Mann und Frau, Erziehung und Bildung ging, sondern konzentrierte sich in einer bemerkenswerten Engführung auf Vorgaben

22 Ingo Resch (Hrsg.), Mehr als man glaubt. Christliche Fundamente in Recht, Wirtschaft und Gesellschaft. Gräfelfing 2000.

23 Martin Niemöller, Lieber russische Diktatur als Dauerspaltung, in: Die Neue Zeitung, 17.12.1949, 2. 
zur Sexualmoral: kein Sex vor der Ehe, das Verbot von bikonfessionellen Ehen, Sexualität gebunden vor allem an den Akt der Zeugung von Kindern.

Wie in wenigen anderen Bereichen manifestierte sich diese Diskursmacht der katholischen Kirche in den öffentlich-rechtlichen Rundfunkanstalten selbst. Die „hinkende Trennung“ zwischen Staat und Kirche bescherte den Kirchenvertretern strukturell und organisatorisch einen starken Einfluss. Über Posten in den Gremien der Rundfunkanstalten waren beide Religionsgemeinschaften einflussreich vertreten. In den thematisch einschlägigen Redaktionen hatten von den Kirchen bestellte Rundfunkbeauftragte die Möglichkeit, unliebsame Berichte mit einem Veto zu stoppen. Die Redaktionen waren sehr kirchennah, beglaubigten das Religiöse publizistisch und verstanden sich als Sprachrohr der Kirchen. Dementsprechend etablierte sich die Bezeichnung „Kirchenfunk“ ${ }^{24}$ Seit Mitte der 196oer Jahre begann sich diese Situation zu verändern: Aus dem „Kirchenfunk“ entwickelte sich der Religionsjournalismus zu einer kritischen Instanz, die nicht nur die Kirchenhierarchie kritisch beobachtete, sondern auch Themen aufbrachte und "nicht davor zurückschreckte, Tabugrenzen aufzubrechen“. ${ }^{25}$ Die Medien spiegelten und beförderten seit den 1970er Jahren die zunehmende Pluralisierung und Politisierung, die auch im religiösen Feld selbst festzustellen war.

Damit stehen die Medienstrukturen paradigmatisch für die Situation insgesamt. Die Diskursmacht der katholischen Kirche aus den 195oer Jahren geriet zunehmend unter Druck. Besonders stark manifestierte sich diese Veränderung auf dem Feld der Sexualmoral. Die Form der autoritären Verkündigung einer zunächst deutlich konfessionell geprägten Vorstellung von Ehe- und Familienpraktiken wurde abgelöst von einem Diskussionsfeld, in dem sich vielfältige Stimmen artikulierten. Die Positionen der kirchlich vorgetragenen Sexualmoral waren spätestens seit Mitte der 196oer Jahre nicht einmal mehr unter Katholikinnen und Katholiken plausibel zu machen. Die Auseinandersetzungen über das schon rasch als „Pillenenzyklika“ etikettierte päpstliche Lehrschreiben „Humanae vitae“ 1968 demonstrierten das aufs Treffendste. ${ }^{26}$

An die Stelle von exkludierenden traten nun zunehmend inkludierende Sprachmuster und Verfahren. Diese Öffnung zu mehr Partizipation bot prinzipiell die Chance, einen größeren Kreis von Personen für die eigene

24 Siehe hierzu und im Folgenden Nicolai Hannig, Die Religion der Öffentlichkeit. Kirche, Religion und Medien in der Bundesrepublik 1945-198o. Göttingen 2O10, 42-103.

25 Ebd., 39o.

26 Birgit Aschmann/Wilhelm Damberg (Hrsg.), Liebe und tu, was du willst? Die „Pillenenzyklika“ Humanae vitae von 1968 und ihre Folgen. Paderborn 2021. 
Position zu gewinnen. Das idealtypisch „absolute Verstehen“ schloss aber faktisch Negationsmöglichkeiten ein und machte vormals als unumstößlich geltende Positionen zum Gegenstand von Verhandlungen, bei denen kein Interesse von vornherein ausgeschlossen werden konnte. Diesem Rechtfertigungsdruck war die kirchliche Sexualmoral nicht gewachsen. Seit den sechziger und siebziger Jahren hat die katholische Kirche ihre begrenzten, aber durchaus wirkungsmächtigen Einflussmöglichkeiten auf die Normierung sexuellen Verhaltens verloren. Ein Zurück zu der engen Verknüpfung von gesellschaftlicher Moral und katholisch geprägter Sexuallehre, wie es in den 1950er Jahren zu beobachten war, scheint seitdem undenkbar. Leben im Glauben umfasst mehr als nur Familienkonzepte und Sexualität, benannte doch die Religion für fast alle Lebensbereiche Regeln und Hinweise. Und dennoch ist damit exemplarisch ein zentrales Moment benannt: Der Bereich von Ehe, Partnerschaft und Sexualität war derjenige, der besonders mit christlichen Moralvorstellungen assoziiert wurde. Die Sexualmoral avancierte in den Nachkriegsjahrzehnten zum Markenkern katholischer Verkündigung.

Gesellschaftlich verlief der Trend völlig gegenläufig: Der Einflussbereich von Religion wurde sukzessive, aber kontinuierlich verschoben und führte zu einer Profanisierung. Ein bislang nicht oder allenfalls nicht-öffentliches Zuwiderhandeln gegen die religiösen Normen wurde nach und nach zum öffentlichen Normalverhalten. Alltagskultur und Lebenswelt emanzipierten sich zunehmend von religiös-konfessionellen Prägungen. Lediglich als Behauptung von Autorität vonseiten der katholischen Kirche selbst wie auch als Stoff für zahlreiche Medienskandale blieb die enge Verbindung von Partnerschaft und Sexualität mit kirchlich-konfessionellen Doktrinen noch weit über die 196oer Jahre hinaus virulent. Mit dem Wegfall der katholischen Deutungsmacht über den Lebensbereich der Sexualität öffnete sich auch der Diskursraum, der dann vor allem ab 2010 eine Skandalisierung von Missbrauch möglich machte.

Die Religionsgemeinschaft, die nach 1945 als „Siegerin in Trümmern“ mit einem enormen Selbstbewusstsein startete, die im Grundgesetz wie auch in den Länderverfassungen dicht eingebunden wurde in staatliche Belange, die sich über eine starke Nähe zur CDU auch parteipolitisch unmittelbar an der Seite der ewigen Kanzlerpartei der Adenauerrepublik befand - diese Religionsgemeinschaft verlor massiv an Einfluss: Neben der stärker werdenden Säkularisierung, die sich vor allem in einer sinkenden Mitgliederzahl, aber auch einer schwindenden religiösen Praxis von Katholikinnen und Katholiken zeigte, wurde auch die so starke Stellung im öffentlichen Diskurs immer stärker hinterfragt. Wie viel kirchlicher Einfluss war notwendig oder, um von der anderen Seite zu fragen, statthaft? Die Wahlhirtenbriefe der katholischen Bischöfe mit ihrer Empfehlung für eine Stimmabgabe zugunsten der CDU waren in der 
Ära Adenauer gängige Praxis, während sie 15 Jahre später als Skandal galten. Damit ist mit einem Beispiel angedeutet, wie stark sich das Empfinden für die Legitimität religiöser Positionen in der säkularen Kultur änderte. Was in den 195oer Jahren noch als moralische Wegmarke galt, war zwei Jahrzehnte später nicht nur eine Position unter vielen, sondern vor allem ein Standpunkt, der sich besonders stark der Kritik zu stellen hatte.

Heute hat sich die damalige Diskursmacht weitgehend aufgelöst, partiell sogar in ihr Gegenteil verkehrt. Während die katholische Kirche vor etwa 70 Jahren noch mit großer Selbstverständlichkeit von vielen Menschen als moralische Instanz gesehen wurde, ist der Vertrauensverlust insbesondere in den letzten Jahren enorm. Der Anteil derjenigen, die die katholische Kirche als eine Institution sehen, der sie ein besonderes Vertrauen entgegenbringen, liegt laut einer Meinungsumfrage vom Beginn des Jahres 2021 bei 15 Prozent. Zum Vergleich: 36 Prozent der Befragten sprechen der evangelischen Kirche eine besondere Vertrauensstellung zu. ${ }^{27}$ Dass mit sinkendem Vertrauen auch ein Machtverlust verbunden ist, liegt auf der Hand. Es ist genau dieser Machtverlust, der auch den Raum eröffnet, über Missbrauch öffentlich zu sprechen und ihn zu skandalisieren.

Dass aktuell insbesondere die katholische Kirche so stark im Mittelpunkt der Diskussion um sexuellen Missbrauch steht, erklärt sich auch über strukturelle Besonderheiten dieser Religionsgemeinschaft. Dies zeigt der historische Vergleich mit der NS-Aufarbeitungsdiskussion der 196oer Jahre. Verhalten begann die bundesdeutsche Gesellschaft, sich an ihrer nationalsozialistischen Vergangenheit abzuarbeiten. Zum bevorzugten Objekt dieser Auseinandersetzung - das hat zuletzt Mark Ruff in seiner Studie gezeigt ${ }^{28}$ avancierte dabei die katholische Kirche. Es waren verschiedene Faktoren, die über die faktische Verstrickung hinaus gerade sie dafür prädestinierten: Ein hohes Selbstideal prägte sie, die sich in ihrer eigenen Geschichtspolitik als eine Institution präsentierte, die dem Nationalsozialismus widerstanden habe. Strikt hierarchisch gegliedert steht mit dem Papst eine Zentralfigur an der Spitze, die eben wegen ihrer herausgehobenen Stellung eine perfekte Zielscheibe bot. Eine vor Selbstbewusstsein strotzende Männergesellschaft hielt an ihrer überkommenen Selbstdarstellung und ihren Ritualen fest. All das bot eine Projektionsfläche, gegen die sich vonseiten derjenigen, die nun auf eine stärkere Auseinandersetzung mit der Diktatur drängten, hervorragend und mit

27 Pressemitteilung RTL/ntv-Trendbarometer: Forsa-Aktuell: Institutionen-Ranking 2021, 11.1.2021, einzusehen unter https://www.presseportal.de/pm/72183/4808331, zuletzt aufgerufen am 7.4.2021.

28 Mark E. Ruff, The Battle for the Catholic Past in Germany, 1945-198o. Cambridge 2017. 
großem Effekt anrennen ließ. Die Schulddiskussion nahm seit der Mitte der sechziger Jahre am Objekt katholische Kirche ihren Anfang, bevor sie dann um 1970 breitere Kreise zog. Auch im Fall der Thematisierung und der Erforschung von Missbrauch ist daher zu berücksichtigen, dass sich in mancher Hinsicht die Gesellschaft stellvertretend auseinandersetzt mit der katholischen Kirche, die sich mit ihrer patriarchalen Grundstruktur, dem Zölibat und einer rigiden Sexualmoral von vielen anderen Zusammenhängen unterscheidet.

\section{Pastoralmacht und Klerikalismus: „Seelenführung“ und der „katholische Geschmack“ des Missbrauchs}

Die Grenzen des Sagbaren sind im katholischen Zusammenhang anders gezogen als beispielsweise im Sportverein, in der Schulklasse oder in der Familie. „Gott freut sich daran, dass wir uns so liebhaben“ - so erklärte und verklärte in der Erinnerung eines Betroffenen der missbrauchende Geistliche seine Gewalttaten. Noch häufiger ist die negative Sanktionierung des vermeintlichen Verrats des Missbrauchs, wenn, wie oben eingeführt, der Pfarrer dem Betroffenen mit "Satan“ droht, falls dieser nicht Stillschweigen bewahrt. Eine solche Absicherung von Missbrauch stützt sich qua Transzendenz auf eine Autorität, die für das gläubige Opfer stärker ist als diejenige des Trainers, des Lehrers oder des Familienvaters. Zugespitzt gilt dann: Im katholischen Zusammenhang wurden die Betroffenen zum Opfer nicht obwohl, sondern weil sie katholisch waren und Überzeugungen mit dem Täter teilten. Die Macht des Täters über sein Opfer rührte aus den spirituellen Zusammenhängen, in die beide eingebunden waren.

Von katholisch höchster Stelle hat Papst Franziskus in seinem Schreiben „an das Volk Gottes" vom 20. August 2018 Klerikalismus als eine der Hauptursachen für den Missbrauch bezeichnet und diesen als „Hochmut" und "Selbstherrlichkeit“ von Priestern beschrieben. „Zum Missbrauch Nein zu sagen, heißt zu jeder Form von Klerikalismus mit Nachdruck Nein zu sagen. ${ }^{\text {} 29}$

So energisch diese Aufforderung auch klingt, so sehr verfehlt sie doch in ihrer Allgemeinheit das katholische Spezifikum von Klerikalismus. Diese Haltung ist nicht allein durch persönliches Fehlverhalten oder Charakterschwächen einzelner Priester oder den Standesdünkel der Geweihten zu erklären. Im Laufe ihrer neuzeitlichen Geschichte hat insbesondere die katholische Kirche

29 Franziskus, Schreiben von Papst Franziskus an das Volk Gottes, 20.8.2018, einzusehen unter https://www.vatican.va/content/francesco/de/letters/2018/documents/papafrancesco_2018082o_lettera-popolo-didio.html, zuletzt aufgerufen am 6.4.2021. 
eine Form von „Pastoralmacht“ entwickelt, die zumindest idealtypisch die nahezu unbeschränkte Machtausübung des Priesters über den Gläubigen systematisch ausgebaut hat. Michel Foucault hat diesen Zusammenhang für beide Kirchen/die katholische Kirche wie folgt beschrieben.

„Die christliche Pastoral bzw. die christliche Kirche [...] hat die einzigartige und der antiken Kultur wohl gänzlich fremde Idee entwickelt, daß jedes Individuum unabhängig von seinem Alter, von seiner Stellung sein ganzes Leben hindurch und bis ins Detail seiner Aktionen hinein regiert werden müsse und sich regieren lassen müsse; daß es sich zu seinem Heil lenken lassen müsse und zwar von jemandem, mit dem es in einem umfassenden und zugleich peniblen Gehorsamsverhältnis verbunden sei.“30

Das christliche „Pastorat“ zielt dieser Konzeption nach auf das Seelenheil jedes Einzelnen und ist deswegen auf eine Totalität zwischen Machtausübendem und dem Übermächtigten angewiesen: Der mächtige Beichtvater wird zum Allwissenden, der mit Gewissensleitung und Gewissenprüfung, Geständnispraxis und der Forderung des reinen Gehorsams eine neue Form der Gouvernementalität ausübt. „Man kann diese Form von Macht nicht ausüben“, so Foucault, „ohne zu wissen, was in den Köpfen der Leute vor sich geht, ohne ihre Seelen zu erforschen, ohne sie zu veranlassen, ihre innersten Geheimnisse zu offenbaren. Sie impliziert Kenntnis des Gewissens und eine Fähigkeit, es zu steuern.“31

In der von Foucault formulierten Absolutheit ist das theoretische Konzept der „Pastoralmacht" statisch, voll von Vorannahmen und somit letztlich für eine historische Analyse kaum nutzbar. Doch weil es in der katholischen Pastoral eine direkte Entsprechung findet, lässt sich mit diesem Konzept dennoch ein wichtiges Spezifikum beschreiben. Übersetzt in die religiöse Sprache der 195oer Jahre, auf viel ältere Wurzeln zurückgehend und nachklingend bis heute - und dabei ist nur vom Normativen, nicht von der Praxis zu sprechen - findet sich Pastoralmacht in der im Katholizismus definierten Aufgabe der "Seelenführung“ wieder. Damit wird eine Form der geistlichen Anleitung bezeichnet, in der der Priester den Gläubigen in seinem religiösen Weg begleitet. Voraussetzung dafür ist, dass der Gläubige nicht nur Sünden und Fehler bekennt, sondern sein ganzes Innenleben mit dem Beichtvater teilt. Was der katholische Theologe Josef Adloff zu Beginn des 20. Jahrhunderts in Büchern wie "Beichtvater und Seelenführer" oder "Seelenführung und

30 Michel Foucault, Warum ich Macht untersuche? Die Frage des Subjekts, in: Hubert L. Dreyfus/Paul Rabinow, Michel Foucault. Jenseits von Strukturalismus und Hermeneutik. Frankfurt a. M. 1987, 243-25o, hier 248.

Ebd. 
Berufspflege" entwickelte, ${ }^{32}$ ist bis heute ein Kernkonzept der priesterlichkatholischen Pastoral. Darin werden zwei Akteursrollen definiert: die des sich vollständig öffnenden Gläubigen wie auch die des sich in seiner Aufgabe verzehrenden, leitenden Geistlichen, der in dieser Position vollkommen überhöht wird.

Dass diese Konzeption keinesfalls als überholt gilt, zeigt der Blick in aktuelle seelsorgliche Literatur und Instruktionen. Die Arbeitshilfe für Beichtväter und Geistliche Begleiter vom 9. März 2011, die die Kongregation für den Klerus veröffentlicht hat, ${ }^{33}$ zitierte Johannes Paul II. und sein nachsynodales Apostolisches Schreiben „Pastores dabo vobis“ (Hirten gebe ich Euch) vom 25. März 1992:

„Die Priester sind in der Kirche und für die Kirche eine sakramentale Vergegenwärtigung Jesu Christi, des Hauptes und Hirten; sie verkünden mit Vollmacht sein Wort, sie wiederholen sein vergebendes Wirken und sein umfassendes Heilsangebot, vor allem durch die Taufe, die Buße und die Eucharistie, sie sorgen wie er liebevoll bis zur völligen Selbsthingabe für die Herde, die sie in der Einheit sammeln und durch Christus im Geist zum Vater führen."34

Die Arbeitshilfe verweist außerdem auf den Idealtypen des Beichtvaters und den Patron der Pfarrer, nämlich den Pfarrer von Ars: Jean-Marie Vianney, geboren 1786, war einfältig, lernschwach, nervös und depressiv. Zeitgenössisch galt er zunächst als wenig geeigneter, vielleicht sogar etwas zurückgebliebener Kandidat für das Amt, der sich schwertat mit Studium und Ausbildung, den Spott seiner Mitbrüder und Vorgesetzten zu ertragen hatte, dann aber zum Vorbild eines sich für den Sünder verzehrenden Priesters stilisiert wurde. Er habe, so die Legende, in den letzten 33 Jahren seines Lebens „täglich 10 bis 17 Stunden im Beichtstuhl [verbracht]. Manchmal hörte er so lange die Beichte, bis er ohnmächtig wurde. Im heißen Sommer 1859 war er am Ende seiner Kräfte und empfing noch krank im Bett Menschen, die die Absolution wollten. Am Morgen des 4. August starb er, wurde 45 Jahre später selig- und im Jahr 1925

$32 \quad$ Josef Adloff, Unio Apostolica sacerdotum saecularium diocesis Argentinensis. Straßburg 1907; ders., Beichtvater und Seelenführer. 3. Aufl. Straßburg 1917; ders., Seelenführung und Berufspflege. Straßburg 1918.

33 Kongregation für den Klerus im Pontifikat von Papst Benedikt XVI. (Hrsg.), Der Priester, Diener der göttlichen Barmherzigkeit. Arbeitshilfe für Beichtväter und Geistliche Begleiter. Vatikanstadt 2011.

34 Ebd., 14. Der Bezugstext ist zu finden in Johannes Paul II., Nachsynodales Apostolisches Schreiben. Pastores dabo vobis, 25.3.1992, einzusehen unter http://www.vatican.va/ content/john-paul-ii/de/apost_exhortations/documents/hf_jp-ii_exh_25031992_ pastores-dabo-vobis.html, zuletzt aufgerufen am 13.4.2021. 
heiliggesprochen". ${ }^{35}$ Dieses Lebensbild des Pfarrers von Ars ist bis heute weit verbreitet und damit desjenigen, der mit seinem „täglichen Martyrium“, dem Abnehmen der Beichte, als Priester Vorbild für die Mitglieder seiner Profession sein soll und in der Ausbildung als beispielhafter "Seelenführer" angeführt wird. ${ }^{36}$

Mit der heutigen Praxis des Beichtsakraments haben diese Schilderungen allenfalls am Rande zu tun: Die Individual- oder Ohrenbeichte ist als Sakrament nahezu vollständig verschwunden und hat kollektiven Formen der Buße Platz gemacht oder - so die Regel - ist im Leben der Gläubigen ersatzlos weggefallen. ${ }^{37}$ In den lehramtlichen Texten, aus denen eben zitiert wurde, wird dieser Einbruch registriert, aber lediglich als Schwundstufe beklagt, gegen die man anarbeiten müsse. „Wo immer ein Priester sich zur Abnahme der Beichte bereithält, kommt früher oder später auch ein Beichtender an; und dort, wo der Beichtvater seine Aufnahmebereitschaft in standhafter Geduld beharrlich beweist, stellen sich viele Beichtende ein! ${ }^{\text {“38 }}$ Es bleibt weiterhin das Ideal des Pfarrers als Seelenführer mit all seiner machtdurchwirkten Dialektik.

Im Zentrum dieser und vieler anderer Überlegungen steht die Person des Priesters. Der geweihte Mann wird systematisch als „sakramentale Vergegenwärtigung Christi“ gedacht. „Zwischen einem Priester und einem rechtschaffenen Laien sollte ein Unterschied sein wie zwischen Himmel und Erde“, so zitierte der Münsteraner Bischof Michael Keller Papst Pius X. in einer Ansprache vor Kandidaten für das Priesteramt und markierte damit die Differenz zwischen Laien und Geweihten. ${ }^{39}$ Darin spiegelt sich die theologische und standesethische Formierung und Disziplinierung letzterer. Allerdings werteten die theologische Deutung, die Ausbildung wie auch die Praxis in der Pastoral Priester im 19. und frühen 20. Jahrhundert nicht allein zu entscheidenden "Milieumanagern“ auf. Zugleich wurde mehr und mehr eine „Totalrolle" geschaffen, in der der geweihte Amtsinhaber als homo dei, als Mann Gottes ${ }^{40}$ sowohl in seiner professionellen Tätigkeit wie auch in der persönlichen Lebensführung höchsten Ansprüchen genügen sollte.

35 Agathe Lukassek, Pfarrer von Ars. Vom Bauern zum Beichtvater, 28.2.2018, einzusehen unter https://www.katholisch.de/artikel/78-vom-bauern-zum-beichtvater, zuletzt aufgerufen am 6.4.2021.

$36 \quad$ Kongregation, Priester, 16.

37 Thomas Großbölting, Der verlorene Himmel. Glaube in Deutschland seit 1945. Göttingen 2013, 39 .

$38 \quad$ Kongregation, Priester, 4.

39 Michael Keller, Priesterliche Heiligkeit - Priesterliche Sendung, in: Unsere Seelsorge, 1959, 98-110, hier 107.

40 Ebd., 99 . 
Die sakrale und ethische Überhöhung und die damit verbundenen Selbst- und Fremdzuschreibungen bildeten den Hintergrund der Debatte um die „Priesterkrise“ seit den 196oer Jahren. Die Fallhöhe für diese Statusgruppe war beträchtlich. Angesichts des gesellschaftlichen Wertewandels seit dem Ende des Jahrzehnts und der zunehmenden Infragestellung traditioneller Autoritätsrollen - sowohl gesamtgesellschaftlich als auch binnenkirchlich - verblasste die sakralisierte Amtsperson des „Hochwürden“ zunehmend. ${ }^{41}$ Theologisch entwickelte das Zweite Vatikanum zwischen 1962 und 1965 entscheidende Impulse und fungierte als Produkt und Promotor der beschriebenen Entwicklungen. Die Konzilsväter verzichteten darauf, eine exklusiv juridische Theologie des Klerus zu formulieren. Stattdessen ordneten sie das Priestertum dem Oberbegriff Gottesvolk zu. Stärker noch als die evangelischen Pfarrer und Pfarrerinnen hatte daher der katholische Klerus seit Mitte der 196oer Jahre seine Rolle neu zu definieren. Auf der einen Seite stand die neu formulierte Vorstellung vom gemeinsamen Priestertum aller Gläubigen, die eine deutlich nivellierende Tendenz hatte und die Amtsautorität in Frage stellte. Auf der anderen Seite blieben der Selbstanspruch und zum Teil auch die Erwartung, dass sich ein Priester deutlich von den übrigen Gläubigen unterscheide. Der „Abschied von Hochwürden“, so urteilte ein Laie bereits 1969, war unwiederbringlich eingeleitet. ${ }^{42}$ Der "Spagat“ des Weltpriesters zwischen Mitchrist und hervorgehobener sakraler Amtsperson wurde seit den 196oer Jahren beständig vergrößert und machte den verunsicherten Klerus schon in der Wahrnehmung der Zeitgenossen zum „Konzilsgeschädigten“.43 Den bislang letzten Höhepunkt der Diskussion um die Rolle und das Rollenverständnis des Klerus markierten die Schriften von Eugen Drewermann: Der Paderborner Priester und Psychoanalytiker erstellte nach eigenen Aussagen „das Psychogramm eines Ideals“ und skizzierte den Klerus als gezeichnet von Selbstverleugnung und psychischer Deformierung. ${ }^{44}$

Beide Facetten des Priesterbildes - die psychosoziale Beschreibung als Mangelwesen wie auch die sakrale Überhöhung - bleiben bis heute in der

41 Klaus Große Kracht, ,Elternrecht' und ,Ehenot'. Familienbilder und Wertewandel im westdeutschen Katholizismus der 195oer und 196oer Jahre, Münster 2018, einzusehen unter https://www.uni-muenster.de/imperia/md/content/religion_und_moderne/preprints/ crm_working_pa-per_18_gro_e_kracht.pdf, zuletzt aufgerufen am 13.4.2021; Großbölting, Himmel, 246f.

42 Josef Othmar Zöller, Abschied von Hochwürden. Seelsorger der Zukunft. Frankfurt a. M. 1969 .

43 Otto Bernhard Roegele, Krise oder Wachstum? Zu Gegenwartsfragen des deutschen Katholizismus. Freiburg im Breisgau 1970, 101.

44 Eugen Drewermann, Kleriker. Psychogramm eines Ideals. 3. Aufl. Stuttgart 1992. 
Diskussion um den sexuellen Missbrauch im katholischen Zusammenhang stark präsent.

Um eine psychosoziale Einordnung geht es mit Blick auf die Taten und die Täter selbst. Der Zölibat als geistige Lebensform hat direkt wenig bis keinen Bezug zum Missbrauch, wohl aber indirekt sind unmittelbare Verbindungslinien zu sehen. Es bleibt zu untersuchen, ob der Priesterberuf Männer anzieht, die Fragen und Unsicherheiten in ihrer psychosozialen Entwicklung ausweichen wollen. Dazu schreibt Heribert Prantl, Kolumnist der Süddeutschen Zeitung: „Es hat sich gezeigt, dass viele Priester, die Minderjährige schänden, in ihrer sexuellen Entwicklung auf der Stufe eines 13-jährigen sind. Das lässt sich nicht mit Beten ändern; das verlangt Änderungen im Kern von Theologie, Amtsverständnis und Recht. Die sexuelle Ausbeutung von Wehrlosen ist das Risiko einer zwangszölibatären, autoritären Kirche“. ${ }^{45}$

Wie stark das Bild des überhöhten Klerikers nachwirkt, zeigte sich bis in die 1990er Jahre hinein im Verhalten der Kirchenhierarchie gegenüber den bekanntwerdenden Missbrauchsfällen. Wenn in Personalkonferenzen und damit dem inner circle der Bistumsleitung in katholisch verdruckster Weise die Rede auf sexuellen Missbrauch von Minderjährigen kam, dann dominierte in der Behandlung dieses Falls oftmals ein Motiv: nämlich das der bischöflichen Fürsorge. Objekt dieser Fürsorge war aber nicht der Betroffene, das Opfer, sondern der Beschuldigte beziehungsweise der Täter. Man sah sich verpflichtet, diesem Kleriker und Mitbruder einen Weg zu eröffnen, weiter Priester zu sein. Oberste Priorität hatte, dass das Sakrament der Priesterweihe und damit die besondere Lebensform weiter Geltung habe. Also galt es, den Missbrauch als Zölibatsbruch zu „reparieren“ und auf diese Weise die Situation zu heilen.

Um das aus der Binnenlogik der Beteiligten nachvollziehen zu können, braucht es viel Theologie und spezielles Wissen darum, was ein Sakrament ausmacht und welche Bedeutung diesem, insbesondere der Priesterweihe, im Katholischen zukommt.

Reflektiert man es aber vor dem Hintergrund einer freiheitlichdemokratischen Grund- und Rechtsordnung, verschieben sich die Koordinaten grundlegend. Dann braucht es viel Fantasie und historischen Weitblick, um vergleichbare Mechanismen und Logiken ausmachen zu können. Wer darüber nachdenkt, wann und wo es solche Parallelgesellschaften schon einmal gab oder immer noch gibt, der landet rasch bei Macht- und Herrschaftsstrukturen der Vormoderne oder den Clan- und Ehrvorstellungen der Mafia und anderer internationaler Verbrecherorganisationen. 
Der Blick auf die vergangenen 70 Jahre Bundesrepublik zeigt deutlich, wie die Pastoralmacht in der seelsorglichen Praxis immer weiter zurückgegangen ist, sich an vielen Stellen gar ganz verflüchtigt oder sich sogar ins Gegenteil verkehrt hat. Damit verschieben sich die Grenzen des Sagbaren, nicht ohne aber in Teilbereichen und in spezifischen Segmenten immer noch blockierend zu wirken. Für viele Betroffene stellt die Pastoralmacht des geweihten Priesters weiterhin eine schwierig zu überwindende Hürde dar, um über das ihnen zugefügte Leid zu sprechen und Gerechtigkeit zu verlangen. 